\title{
Hemocultivos: Uso e Indicaciones.
}

\section{Blood cultures: Use and Indications.}

Felipe Andrés Solano Rojas' María Fernanda Valverde Solano² Kevin Josué Oviedo Pérez ${ }^{3}$ Alberto Quesada Pacheco ${ }^{4}$ Carolina Gutiérrez Méndez ${ }^{5}$

1, 2, 3 y 4 Médico general. Caja Costarricense de Seguro Social, San José Costa Rica. 5 Bachiller en Ciencias Médicas Trabajador independiente, San José Costa Rica

Contacto: felipeasolano@gmail.com

\section{Cómo citar:}

Solano Rojas, F. A. Valverde Solano, M. F., Oviedo Pérez, K. J., Quesada Pacheco, A., \& Gutiérrez Méndez, C. Hemocultivos: Uso e Indicaciones. Revista Ciencia Y Salud Integrando Conocimientos, 5(6), Pág. 43-49. https:// doi.org/10.34192/ cienciaysalud.v5i6.366

Recibido: 01/Sep/2021 Aceptado: 25/Nov/2021 Publicado: 03/ene/2022

\section{RESUMEN}

La bacteriemia se ha asociado a una mortalidad considerable en el paciente hospitalizado, por lo que se han desarrollado reglas de decisión clínica para calcular la probabilidad pre-test de tener una bacteriemia; estas no se usan con frecuencia en la práctica clínica, ni en datos clínicos aislados como la fiebre, leucocitosis, taquicardia o aumento de marcadores inflamatorios, que no son útiles. Los hemocultivos son un arma de doble filo en el abordaje del paciente séptico, si bien su utilidad en estos pacientes está establecida, su uso irracional genera un uso inadecuado de antibióticos, un aumento en la media de estancia hospitalaria y los costos hospitalarios. La decisión para realizar la toma de hemocultivos debe ser individualizada en cada paciente, tomando en cuenta predictores de bacteriemia y calculando la probabilidad pre-test de obtener un verdadero positivo, especialmente en pacientes que ya han iniciado un esquema de antibióticos o en quienes ya se ha documentado un agente microbiológico.

Palabras Clave: Hemocultivos, bacteriemia, shock séptico, antibióticos, fiebre.

\section{ABSTRACT}

Bacteremia is associated with increased mortality in the hospitalized patient, hence clinical decision rules have been developed to calculate the pre-test probability of a bacteraemic episode; they are not used frequently in clinical practice and isolated clinical signs like fever, leukocytosis, tachycardia or increase in inflammatory markers are not useful. Blood cultures are a double-edge weapon in the approach of the septic patient, while its usefulness is straightforward, an irrational use leads to a poor antibiotic use, and to an increase in the median lenght of hospital stay and in hospitalary expenses. The decision to draw blood cultures should be individualized to each patient, assessing for bacteraemia predictors and calculating the pre-test probability of obtaining a true positive, especially in patients who are already receiving antibiotics or in whom a microbiological agent has already been documented.

Keywords: Blood Cultures, bacteremia, septic shock, antibiotics, fever. 


\section{INTRODUCCIÓN}

Se estima que hay más de 200000 episodios de bacteriemia al año (1), y cada episodio documentado se asocia a una mortalidad de $14-37 \%$ en salón general $(1,2)$. Actualmente, la habilidad para predecir estos episodios de bacteriemia es baja, de todos los hemocultivos tomados en pacientes hospitalizados, solamente el 5 a 10\% son positivos; y de estos, 30 a 50\% son contaminantes (2, 3). El estudio Diagnostic Stewardship Intervention To Improve Blood Culture Use among Adult Nonneutropenic Inpatients (DISTRIBUTE) (3) demostró que el uso liberal de hemocultivos aumenta el riesgo de obtener falsos positivos, lo cual extiende los días de estancia hospitalaria, el uso de antibióticos, el número de exámenes innecesarios y los costos hospitalarios. Además, cuando se obtienen verdaderos positivos, algunos médicos se muestran resistentes a cambiar el esquema de antibioticoterapia a uno con menor amplitud (4).

Algunos estudios indican que una gran cantidad de hemocultivos se realiza de forma innecesaria, aumentando los costos y el riesgo de hemocultivos contaminados $(5,6)$, por ejemplo: se estima que el $30 \%$ de todos los hemocultivos realizados en Unidades de Cuidados Intensivos ( $\mathrm{UCl}$ ) médicos y más del $40 \%$ de los hemocultivos realizados en servicios de medicina son inapropiados, usualmente indicados por procesos no infecciosos, fiebre persistente, cultivos de seguimientos o en pacientes con bajo riesgo de bacteriemia (3).

En UCl, la sospecha clínica de sepsis se tiene en alrededor de un 9\% de los pacientes, con una mortalidad de hasta $60 \%$, sin embargo, se documenta un agente microbiológico solamente en un $71 \%$ de los casos, siendo las bacteriemias alrededor de 53\% (7). Muchos pacientes ya han recibido antibioticoterapia de manera empírica previo a su ingreso a la $\mathrm{UCl}$, disminuyendo el rendimiento de los cultivos $(1,7)$, de los cuales: un $50 \%$ de los positivos es por contaminantes, aumentando en un $39 \%$ el uso de antibióticos $(2,3,7)$.

La eficiencia de los hemocultivos en pacientes hospitalizados suele ser bastante baja, y los cultivos tomados "de rutina" suelen tener poco valor clínico (8).

\section{METODOS}

Para la elaboración de este artículo se realizó una búsqueda en la base de datos de PubMed con las palabras: blood cultures, sepsis, antibiotics, guidelines, hemocultivos, contaminantes, febril. Se seleccionaron artículos en inglés y en español relevantes al tema central de investigación, debido a la falta de bibliografía afín al tema principal, se incluyeron estudios publicados máximo hasta el año 2000.

\section{El paciente febril:}

La fiebre aislada no es un predictor adecuado de bacteriemia $(3,4)$, es frecuente la toma de hemocultivos de manera refleja ante la fiebre, lo cual aumenta el riesgo de contaminantes y aumenta los costos (3, 8), además, la bacteriemia suele iniciar 1-2 horas antes del pico febril, lo cual es difícil de predecir en el momento, por lo que el tiempo en que se toman los hemocultivos no es tan importante (9).

Se pensaba que el fenómeno de la fiebre precedida por una bacteriemia se debía a bacteriemias intermitentes, sin embargo, esto no tiene adecuada evidencia y no debería ser un justificante para realizar múltiples hemocultivos (10). Existe ahora el concepto de bacteriemias continuas con una densidad muy baja de microorganismos ( $7,9,10)$, tomando en cuenta esto, y además como se ha documentado en múltiples estudios $(8,9,10,11)$ el determinante más importante en la eficiencia de un hemocultivo es la cantidad de sangre enviada a cultivar y no el momento en el cual se toma el hemocultivo. Enviar 30 - $40 \mathrm{~mL}$ de sangre aumenta el rendimiento entre 4,2 a $19 \%(10,11)$.

Realizar hemocultivos de control por fiebre aislada indiferenciada no tiene valor clínico, salvo en casos de endocarditis infecciosa o bacteriemia documentada por S. aureus. (1, 3). 


\section{CIENCIA\&SALUD}

\section{El paciente persistentemente febril:}

En pacientes hospitalizados infectados, es frecuente tener fiebre persistente, aun cuando se ha iniciado antibioticoterapia de manera empírica, Grace et. al. (1) en su estudio retrospectivo para determinar la eficiencia de los cultivos tomados 72 horas posterior a inicio de antibióticos, demostraron que ni la edad, sexo, temperatura, conteo de leucocitos o comorbilidades, eran predictores de aislamiento microbiano en estos pacientes. En su análisis, solamente la presencia de endocarditis o el aislamiento de Staphylococcus aureus (S. aureus) en cultivos pre antibiótico, predijeron el aislamiento en cultivos subsecuentes posterior a inicio de antibióticos. Los autores de este estudio sugieren que una vez que se ha identificado un microorganismo, realizar cultivos de seguimiento no es necesario, excepto en pacientes con bacteriemia por S. aureus. El estudio DISTRIBUTE (3) extiende esta recomendación también para pacientes con sospecha de endocarditis y además a pacientes con riesgo de infección asociada a un catéter intravascular, lo cual ha demostrado ser un predictor de bacteriemia también en otros estudios $(5,7,12)$.

En el caso de la fiebre persistente y la toma de hemocultivos seriados, Shafazand et. al. (7) estimaron que, en casos de bacteriemia, la probabilidad de tener un segundo set de hemocultivos positivos es de $85 \%$, ascendiendo a más de 95\% en casos de endocarditis, si el primer set ha dado positivo. Esto concuerda con estudios previos de Gross, et. al. (1) quienes recomiendan no realizar hemocultivos de seguimiento en casos de fiebre persistente cuando no se logra documentar un agente microbiológico en el primer set de cultivo.

\section{Hemocultivos seriados:}

En el manejo de bacteriemias, los hemocultivos seriados positivos se asocian a un aumento en la mortalidad (5), por lo cual se podría intuir que realizar hemocultivos de control en pacientes con bacteriemia ya documentada podría tener un valor clínico, sin embargo Wiggers, et. al. (5) en su estudio retrospectivo de cohorte, evaluaron 2715 pacientes con hemocultivos positivos, de los cuales 914 (33,7\%) fueron contaminantes y solamente 118 pacientes se documentaron con bacteriemia persistente (6,6\%), los autores mencionan que solamente tres pacientes en el grupo de bacteriemia persistente tuvieron un cambio en su esquema de antibióticos. En este estudio los predictores independientes de bacteriemia persistente fueron: sexo masculino (OR (odds ratio) 2.59, IC 95\% 1.28 - 5.25), admisión a un servicio de medicina (OR 2.80, IC 1.34 - 5.84), bacteriemia por S. aureus (OR 4.99; IC 95\% 1.88 - 10.73), foco de infección endovascular (OR 7.66; IC 95\% 2.30 - 25.48) o epidural (OR 26.99; IC 1.91 - 391.08).

Canzoneri, et. al. (6) en su estudio sobre la necesidad de realizar hemocultivos de control en pacientes con bacteriemia por bacilos gram negativos, analizaron 500 episodios de bacteriemia y documentaron que al contrario de estudios anteriores: al realizar cultivos de seguimiento, la fiebre en el día en que se tomó el hemocultivo de control era un factor predictivo independiente de bacteriemia persistente, sin embargo en su estudio no hubo ninguna asociación entre los hemocultivos positivos de control y un aumento en la mortalidad o en el número de admisiones a unidades de cuidados intensivos. Los autores concuerdan con que realizar hemocultivos de manera liberal aumenta el riesgo de contaminación, los costos hospitalarios, la estancia hospitalaria y el uso inapropiado de antibióticos.

En pacientes hospitalizados infectados, con un germen ya identificado, la presencia de fiebre persistente no aumenta la mortalidad $(5,6)$, y se estima que solamente un $0.72 \%$ de todas las bacteriemias captadas en hemocultivos seriados en estos pacientes, se debe a un agente microbiano distinto al identificado inicialmente (5), tomando en cuenta que los hemocultivos seriados aumentan el riesgo de falsos positivos, no se puede recomendar esta práctica en todos los casos de bacteriemia; se recomienda únicamente en casos específicos donde hay una muy alta probabilidad de bacteriemia persistente y donde su documentación puede tener un impacto en la terapéutica (endocarditis infecciosa, infección endovascular, bacteriemia por S. aureus, candidemia) $(1,3,5,8,12)$. 


\section{Predictores de bacteriemia:}

Si bien los estudios son heterogéneos en cuanto a las variables que determinan la probabilidad de encontrar una bacteriemia real, algunos concuerdan $(2,3,5,6)$ en que entre los predictores de bacteriemia se encuentra la sospecha de: endocarditis infecciosa, infecciones endovasculares, infecciones asociadas a catéteres, además de datos sugestivos de daño orgánico (e.j PAS < 90mmHg, lesión renal aguda, coagulación intravascular diseminada, hiperlactatemia) $(4,12)$ y la incapacidad para realizar un adecuado control de la fuente infecciosa (5).

En el estudio de Coburn, et. al. (12) se realizó una revisión de 35 estudios para determinar la prevalencia y probabilidad de encontrar una bacteriemia, ellos documentaron que una temperatura por encima de $38^{\circ} \mathrm{C}$ tenía un OR de 1.9 (IC 95\%, 1.4-2.4) de tener una bacteriemia, sin embargo la evaluación de los estudios fue muy heterogénea, estos autores mencionan que los escalofríos en pacientes febriles parecen tener una mayor utilidad para identificar episodios de bacteriemia, con un OR de 2.2 (IC 95\%, 1.4-3.3), de igual forma en su estudio la presencia de hipotensión, definida como una presión arterial sistólica (PAS) menor a $90 \mathrm{mmHg}$ o la necesidad de utilizar vasopresores, así como un conteo de plaquetas menor de 150000/uL, linfopenia menor de 500/uL o un índice de neutrófilos-linfocitos mayor a 10, aumentaban la probabilidad de bacteriemia. Estos datos clínicos son sugestivos de sepsis severa y daño orgánico (13), lo cual nos debe llevar a considerar el contexto del paciente y no debería sugerir la necesidad de hemocultivos basados únicamente en estos datos de manera aislada.

Con respecto al síndrome de respuesta inflamatoria sistémica (SIRS), el cual se ha documentado como un predictor de bacteriemia (12), se debe recordar que en pacientes quirúrgicos particularmente, el trauma tisular y el estrés fisiológico post operatorio pueden generar leucocitosis, fiebre y datos de SIRS (5). Coburn, et. al. (12) mencionan que la probabilidad de bacteriemia para pacientes con meningitis, shock séptico y sepsis severa es de 38-69\%, además de otros signos ya mencionados los cuales son indicadores de disfunción orgánica, esto podría implicar que la severidad del cuadro clínico podría ser un mejor predictor de bacteriemias y de hemocultivos con un verdadero positivo, se desconoce si se ha realizado un estudio que compruebe esta hipótesis.

Algunos autores recomiendan valorar la probabilidad pre-test para obtener un hemocultivo positivo antes de indicarlo $(4,7,12)$; Shapiro, et. al. (4) en su estudio de cohorte prospectivo desarrollaron y validaron una regla de decisión (tabla 1) para determinar la probabilidad de que un paciente en el servicio de emergencias o con al menos tres horas admitido en hospitalización, tenga una bacteriemia. Esta regla agrupa a los pacientes en categorías de riesgo bajo (0-1 puntos), moderado (2-5 puntos) y alto (> 6 puntos), acorde con la cantidad de criterios que cumplan, en cada categoría el riesgo de una bacteriemia es de $0.6 \%, 6.8 \%$ y $25.6 \%$ respectivamente. Presenta una sensibilidad de $98.0 \%$ y especificidad de $29.0 \%$, con un valor predictivo positivo y negativo de $11.1 \%$ y $99.4 \%$ respectivamente, lo cual le confiere un poder adecuado para tomar decisiones, cuando estos criterios se encuentran ausentes es muy poco probable que el paciente tenga una bacteriemia y por tanto la toma de hemocultivos no está indicada. Los autores mencionan que utilizando esta herramienta se puede reducir hasta en un $27 \%$ la toma de hemocultivos, sin embargo, a nuestro conocimiento, esta regla no es utilizada con frecuencia.

Existen marcadores como la procalcitonina que se han descrito

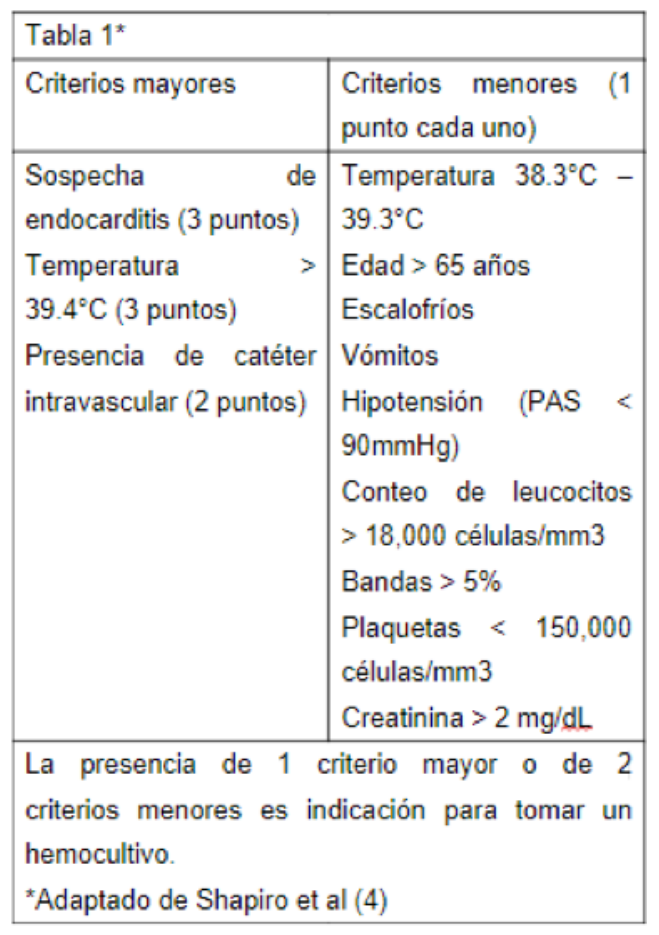


como una herramienta para determinar la probabilidad de que un paciente se encuentre infectado, sin embargo su valor es mayor para descartar la presencia de una bacteriemia que para probar la misma (14). En este sentido, Van Ser Geest, et. al. (15) buscaron determinar el valor de la procalcitonina para la decisión de tomar hemocultivos en pacientes críticos; documentaron que un valor de corte de procalcitonina de $<0.25$ $\mathrm{ng} / \mathrm{mL}$ tiene un valor predictivo negativo y positivo de $96 \%$ y $29 \%$ respectivamente, con una sensibilidad de $98 \%$ y una especificidad de $20 \%$; mencionan que de utilizarse la procalcitonina como un predictor pre-test se pudo haber reducido en un $17 \%$ la toma de hemocultivos. Esto concuerda con el estudio de Savitri, et. al. (15). La procalcitonina se puede utilizar como un marcador para descartar la posibilidad de bacteriemia, sin embargo, se considera que la toma de decisiones debe realizarse valorando todo el cuadro clínico y no con un valor aislado de laboratorio.

\section{Hemocultivos en el paciente con antibióticos:}

El uso de antibióticos de manera temprana en el paciente séptico es una de las medidas con reducción de mortalidad más importantes en el manejo de estos pacientes $(12,13,16,17)$. En pacientes hospitalizados se puede encontrar ante la decisión de tomar un hemocultivo en pacientes que ya han iniciado esquema de antibióticos o que ya tienen un aislamiento microbiológico, al respecto: en un estudio retrospectivo donde evaluaron la toma de hemocultivos antes del inicio de antibióticos y tras 72 horas de iniciarlos, en 149 pacientes febriles $(1,7)$, determinaron que de los 56 cultivos positivos iniciales que obtuvieron, solamente 26 continuaban persistentemente positivos posterior a 72 horas de antibioticoterapia, y de estos solamente en un paciente se aisló un microorganismo distinto. Esto concuerda con el racional de no realizar cultivos seriados de control como fue comentado anteriormente.

El $30 \%$ de todos los hemocultivos que se realizan se indican de manera innecesaria y sobre todo en pacientes en quienes ya se han iniciado antibióticos (3), tomando en cuenta que el 91.5\% de los episodios de bacteriemias que son detectadas se documentan con el primer set de hemocultivos (7) y que el uso liberal de antibióticos, sobre todo en pacientes que no lo requieren, puede generar: toxicidad asociada a los antimicrobianos, toxicidad mitocondrial, reacciones adversas a medicamentos, selección selectiva de organismos resistentes y disrupción del microbioma del paciente (18), se debe realizar una valoración minuciosa e individualizada a la hora de considerar la toma de hemocultivos en estos pacientes, sobre todo en casos en que ya se ha documentado un agente microbiológico.

\section{CONCLUSIONES}

El uso liberal de hemocultivos aumenta el riesgo de obtener falsos positivos, lo cual aumenta los días de estancia hospitalaria, el número de exámenes innecesarios y el uso inadecuado de antibióticos.

La fiebre aislada no corresponde a un predictor fiable de bacteriemia y no se debe recomendar el uso de hemocultivos únicamente por un episodio febril. Es frecuente documentar fiebre persistente durante las primeras 72 horas de inicio de antibioticoterapia, sin embargo, solamente está recomendado la toma de hemocultivos seriados en pacientes con endocarditis infecciosa, candidemia o bacteriemia por S. aureus.

Pocos modelos de predicción de bacteriemia han sido validados, y estos no suelen ser utilizados en la práctica clínica, sin embargo, el criterio clínico es un mal predictor de bacteriemia, particularmente cuando se realizan hemocultivos de manera refleja ante un episodio febril.

Las reglas de decisión clínica que se han desarrollado parecen ser útiles para valorar la probabilidad pretest de una bacteriemia, al igual que marcadores como la procalcitonina o datos clínicos de SIRS, tienen una sensibilidad para determinar, en su ausencia, cuál paciente tiene una probabilidad muy baja de tener una bacteriemia.

Signos clínicos aislados como fiebre, leucocitosis, taquicardia no son adecuados predictores de bacteriemia y se debe valorar cada paciente de manera individual para definir la probabilidad pre-test de obtener un verdadero positivo al realizar un hemocultivo. 


\section{REFERENCIAS BIBIOGRÁFICAS}

1) Grace C, Lieberman J, Pierce K, Littenberg B. Usefulness of Blood Culture for Hospitalized Patients Who Are Receiving Antibiotic Therapy. Clin Infect Dis. 2001; 32(11):1651-1655.

2) Eliakim-Raz N, Bates D, Leibovici L. Predicting bacteraemia in validated models - a systematic review. Clin Microbiol Infect. 2015; 21(4):295-301.

3) Fabre V, Klein E, Salinas A, Jones G, et al. A Diagnostic Stewardship Intervention To Improve Blood Culture Use among Adult Nonneutropenic Inpatients: DISTRIBUTE Study. J Clin Microbiol [Internet]. 2020 [Consultado 29 Jul 2021]; 58(10):e01053-20. Disponible en: http://www.jcm.asm.org.

4) Shapiro N, Wolfe R, Wright W, Moore R, Bates D. Who Needs a Blood Culture? A Prospectively Derived and Validated Prediction Rule. J Emerg Med. 2008; 35(3):255-264.

5) Wiggers J, Xiong Wei, Daneman N. Sending repeat cultures: is there a role in the management of bacteremic episodes? (SCRIBE study). BMC Infect Dis [Internet]. 2016 [Consultado 29 Jul 2021]; 16:286. Disponible en: https://bmcinfectdis.biomedcentral.com.

6) Canzoneri C, Akhavan B, Alcedo P, Aisenberg G. Follow-up Blood Cultures in Gram-Negative Bacteremia: Are They Needed?. Clin Infect Dis. 2017; 65(11):1776-1779.

7) Shafazand S, Weinacker A. Blood Cultures in the Critical Care Unit: Improving Utilization and Yield. Chest. 2002; 122(5):1727-1736.

8) Kirin T, Weinstein M. Update on blood cultures: how to obtain, process, report, and interpret. Clin Microbiol Infect. 2013; 19(6):513-520.

9) Leyssene D, Gardes S, Vilquin P, Flandrois J, Carret G, Lamy B. Species-driven interpretation guidelines in case of a single-sampling strategy for blood culture. Eur J Clin Microbiol Infect Dis. 2011; 30(12): 1537-1541.

10) Dargere S, Parienti J, Roupie E, Gancel P, et al. Unique blood culture for diagnosis of bloodstream infections in emergency departments: a prospective multicentre study. Clin Microbiol Infect. 2014; 20(11):0920-0927.

11) Lamy B, Dargere S, Arendrup M, Parienti J, Tattevin P. How to Optimize the Use of Blood Cultures for the Diagnosis of Bloodstream Infections? A State-of-the Art. Front Microbiol [Internet]. 2016 [Consultado el 29 Jul 2021]; 7:697. Disponible en: https://frontiersin.org.

12) Coburn B, Morris A, Tomlinson G, Detsky A. Does This Adult Patient With Suspected Bacteremia Require Blood Cultures?. JAMA. 2012; 308(5):502-511.

13) Gyawali B, Ramakrishna K, Dhamoon A. Sepsis: The evolution in definition, pathophysiology, and management. SAGE Open Med [Internet]. 2019 [Consultado el 29 Jul 2021]; 7:2050312119835043. Disponible en: https://journals.sagepub.com.

14) Kibe S, Adams K, Barlow G. Diagnostic and prognostic biomarkers of sepsis in critical care. J Antimicrob Chemother. 2011; 66(2): ii33-ii40.

15) Van der Geest P, Mohseni M, Nieboer D, Duran S, Groeneveld A. Procalcitonin to guide taking blood cultures in the intensive care unit; a cluster-randomized controlled trial. Clin Microbiol Infect. 


\section{CIENCIA\&SALUD}

2017; 23(2):86-91.

16) Strich J, Heil E, Masur H. Considerations for Empiric Antimicrobial Therapy in Sepsis and Septic Shock in an Era of Antimicrobial Resistance. J Infect Dis. 2020; 222(2):S119-S131.

17) Weinberger J, Rhee C, Klompas M. A Crítical Analysis of the Literature on Time-to-Antibiotics in Suspected Sepsis. J Infect Dis. 2020; 222(2): S110-S118.

18) Arulkumaran N, Routledge M, Schlebush S, Lipman J, Conway A. Antimicrobial-associated harm in critical care: a narrative review. Intensive Care Med. 2020; 46(2):225-235 